\title{
Effect of Centrally Administered Interleukin-1 and Endotoxin on Food Intake of Fasted Rats
}

\author{
DONNA O. MCCARTHY, MATTHEW J. KLUGER' AND ARTHUR J. VANDER \\ School of Nursing and Department of Physiology. The University of Michigan, Ann Arbor, MI 48109
}

Received 8 July 1985

\begin{abstract}
MCCARTHY, D. O., M. J. KLUGER AND A. J. VANDER. Effect of centrally administered interleukin-l and endotoxin on food intake of fasted rats. PHYSIOL BEHAV 36(4) 745-749, 1986.- We have previously shown that interleukin-1 (IL-1), a polypeptide known to mediate many aspects of the acute phase response to infection, suppresses food intake when injected intraperitoneally into fasted rats. IL-1 acts at the level of the hypothalamus to induce fever. In view of the large number of peptides that have been shown to alter food intake as well as body temperature when injected intracerebroventricularly (ICV), we hypothesized that the receptor site for the anorexigenic activity of IL-1 would be located in a central nervous site bathed by the cerebrospinal fluid. In the present study, ICV injection of IL-1 or E. coli endotoxin (a stimulus for the synthesis of IL-1), significantly elevated body temperature, but did not affect food intake of fasted rats. We conclude that receptors mediating the anorexigenic actions of IL-1 or endotoxin are not located at a central nervous site bathed by the cerebrospinal fluid. Furthermore, fever per se is not reponsible for the reduction in food intake seen following peripheral injection of IL-1 or endotoxin.
\end{abstract}

Fever Anorexia Naloxone $\mathrm{HCl}$ Interleukin-1 Endotoxin

ACUTE infectious illness precipitates a series of stereotypical responses, including fever, granulocytosis, skeletal muscle catabolism, and alterations in plasma levels of iron, zinc, glucoregulatory hormones, and acute phase proteins. Virtually all of these responses are thought to be mediated either directly or indirectly by interleukin-1 (IL-1), a $17.5 \mathrm{kd}$ heat-labile peptide synthesized and released by mononuclear phagocytes in response to infection $[7,17]$. Another common manifestation of acute infectious illness is a decrease in food appetite. We have found that a peripheral injection of IL-1 suppresses food intake of fasted rats to levels approximating those seen following injection of $E$. coli endotoxin [14]. These findings suggest that infection-induced anorexia is, in part, due to the release of IL-1.

The regulation of food intake is a complex phenomenon, involving multiple peripheral and central satiety signals that are integrated at the level of the hypothalamus [15]. Hamilton has proposed that central nervous regulation of food intake is influenced by mechanisms involved with the regulation of body temperature [9]. Indeed, several monoamines and neuropeptides implicated in the central regulation of food intake will alter body temperature when injected into the hypothalamus or cerebral ventricles $[4,6]$. Because IL-1 acts at the level of the anterior hypothalamus to induce an elevation in body temperature during infection, it is possible that IL-1 may act at this same site to decrease food appetite. It is also possible that the reduction in food intake seen fol- lowing injection of endotoxin is not mediated centrally by IL- 1 , but by endotoxin itself $[3,16]$. Comparison of the effects of centrally injected IL-1 or endotoxin on food intake of rats will help to distinguish between these possibilities.

\section{METHOD}

\section{Animals and Experimental Protocol}

Male Sprague-Dawley rats weighing 200 grams were housed individually in a temperature-controlled room at $27 \pm 1{ }^{\circ} \mathrm{C}$, the thermoneutral zone of the rat. Each rat was stereotaxically implanted with a stainless steel cannula (\#220, David Kopf, Tujunga, CA) in the right lateral cerebral ventricle, as described by Bailey et al. [2]. For the measurement of body temperature, each rat was implanted with a temperature sensitive miniature AM transmitter in the peritoneal cavity (Minimitter Co., Sunriver, OR). These minimitters had been calibrated against a temperaturecontrolled water bath immediately prior to implantation. The rats were allowed 7-9 days to recover from the surgical procedures.

To ensure that the rats would eat during an experiment, they were fasted overnight (water was available ad lib). The rats were fed a liquid diet (Lieber-Dicarli 82, Bioserv Inc., Nutley, NJ) to facilitate the frequent measurement of food intake without disturbing the animals during the course of the experiments. The rats were allowed a 48 hour rest period

'Requests for reprints should be addressed to Matthew J. Kluger, Department of Physiology, 7620 Medical Science II, The University of Michigan, Ann Arbor, MI 48109. 

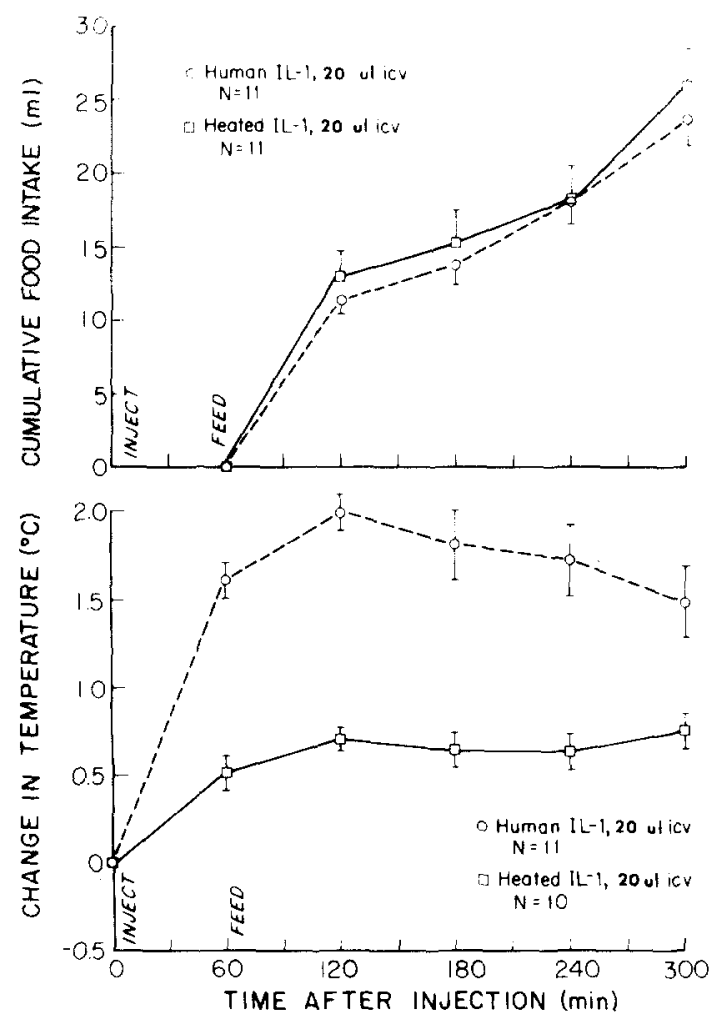

FIG. 1. Cumulative milliliters of food intake $( \pm$ S.E.M.) and change in body temperature $( \pm$ S.E.M.) in fasted rats following intracerebroventricular injection of $20 \mu \mathrm{l}$ human monocyte supernatant containing IL-1 (O) or supernatant heated to $70^{\circ} \mathrm{C}$ for 90 minutes $(\square)$.

between experiments and were maintained on the liquid diet to extinguish any conditioned aversion to the diet resulting from a previous experiment. The animals were conditioned to the feeding schedule and handling for three days prior to the onset of an experiment. At all times, the animals were treated in a manner consistent with the DHEW "Guidelines for the care and use of laboratory animals."

For each experiment, the rats were injected one hour prior to being allowed free access to the liquid food. Milliliters of food intake from the calibrated feeding bottles were measured every 60 minutes for a total of 4 hours and the mean hourly food intake was computed for the four-hour feeding period. Because rats gain about $25 \mathrm{~g}$ body weight per week, with food intake increasing concomitantly, comparisons were made only between the experimental and control groups for each substance injected. Data were analyzed by Student's $t$-test.

Body temperature was monitored to validate activity of IL-1 at the level of the hypothalamus, i.e., onset of fever following injections. Body temperature was measured twice prior to injection and every 60 minutes after injection for a total of 5 hours, inclusive of the four-hour feeding period. Baseline temperatures of the rats were determined by averaging the two readings taken before injection. The change in temperature was determined by subtracting the baseline measurement from subsequent measurements taken after injection. The mean change in temperature was determined by averaging the temperature changes over the five-hour postinjection period.

Whenever baseline temperature exceeded $38^{\circ} \mathrm{C}$, or there
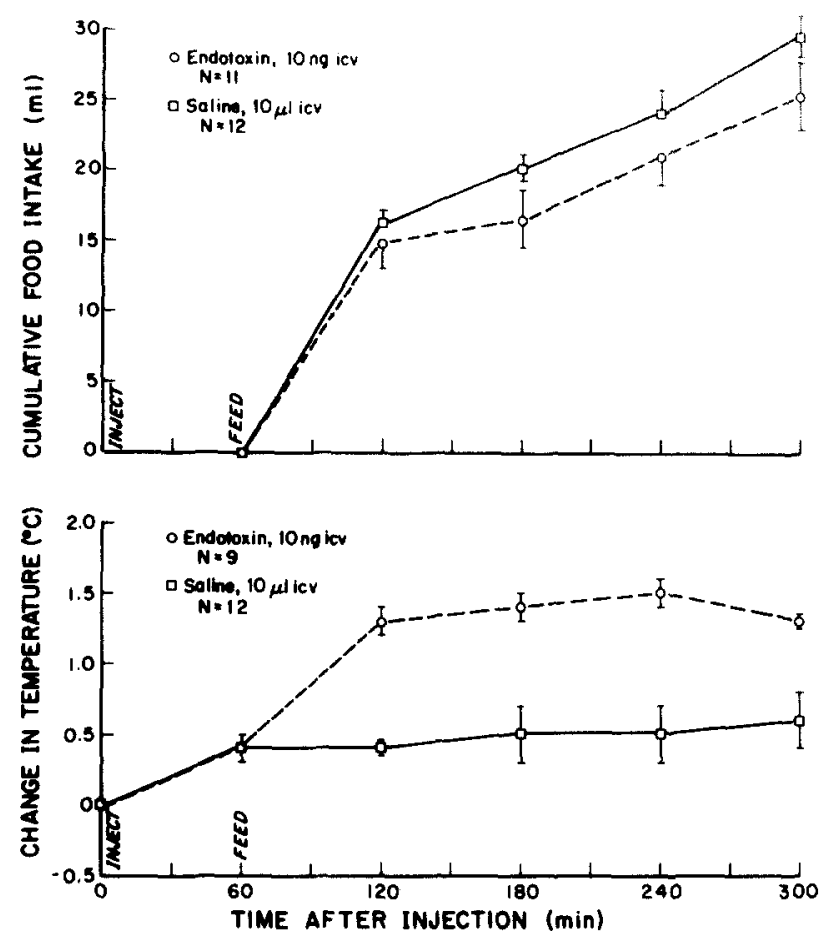

FIG. 2. Cumulative milliliters of food intake ( \pm S.E.M.) and change in body temperature $( \pm S$.E.M. $)$ in fasted rats following intracerebroventricular injection of $10 \mathrm{ng} \mathrm{E}$. coli endotoxin in $10 \mu \mathrm{l}$ saline ( $)$ or $10 \mu \mathrm{l}$ of saline alone $(\square)$.

was any suggestion of leakage or inflammation around the cannula, the animal was eliminated from the experiment. On occasion, a minimitter battery would fail, and this explains why sample sizes for body temperature and food intake data are not always the same in a given experiment. Upon completion of the last experiment, the rats were decapitated and $50 \mu \mathrm{l}$ of trypan blue injected into each cannula. The brain of each animal was dissected to confirm placement of the cannula; data from any animal in whom the ventricular system was not stained was eliminated from anaysis.

\section{Preparation of Interleukin-1}

The preparation of human monocyte supernatant containing IL-1 was based on the method described by Jones $e t$ al. [10]. In brief, $70-90 \mathrm{ml}$ of heparinized blood were obtained from healthy adult human volunteers. Mononuclear cells were separated by density centrifugation on a FicollIsopaque gradient. The mononuclear cells were washed 3 times in Hanks' Balanced Salt Solution (HBSS; Gibco, New York), counted using a hemocytometer and phase microscope, and resuspended in HBSS at a concentration of $2.5 \times 10^{6}$ monocytes $/ \mathrm{ml}$ in a total volume of no greater than 2 $\mathrm{ml}$ in a $12 \times 75$ polypropylene plastic tube (Falcon Co., Oxnard, CA). The cells were stimulated with heat-killed $S$. epidermidis by mixing on a tilt mixer at $37^{\circ} \mathrm{C}$ for 1 hour at a ratio of 20 bacteria/monocyte in the presence of $10 \%$ fresh pooled human serum. After the 1 hour stimulation, the cell suspensions were centrifuged for 15 minutes at $500 \mathrm{~g}$, washed in HBSS, and resuspended in HBSS at a concentra- 

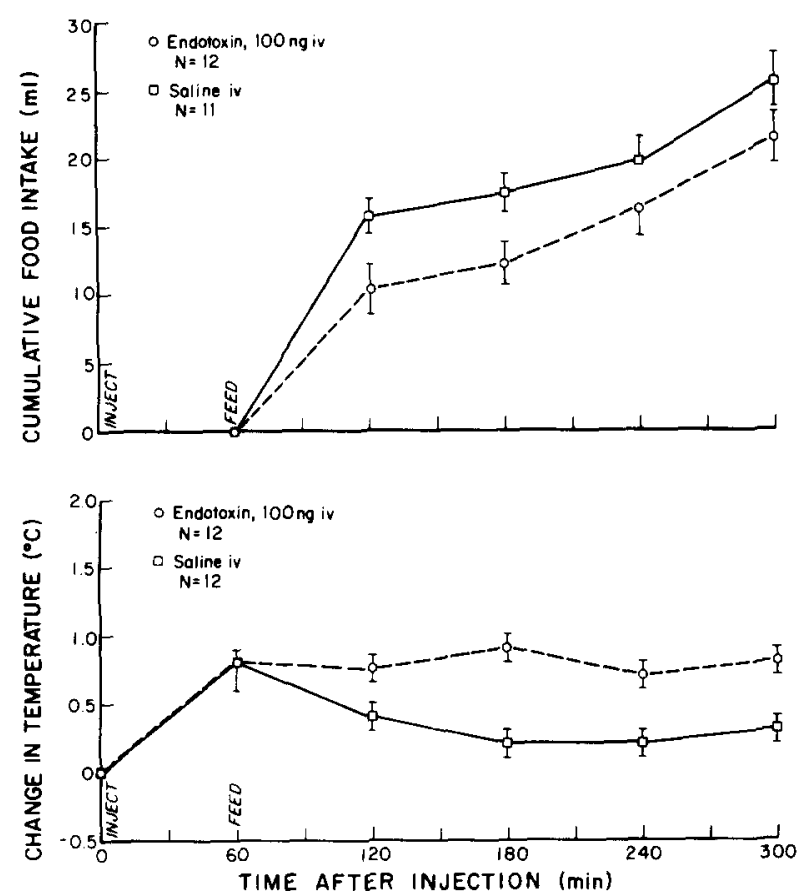

FIG. 3. Cumulative milliliters of food intake $( \pm$ S.E.M.) and change in body temperature $( \pm$ S.E.M. $)$ in fasted rats following intravenous injection of $100 \mathrm{ng} E$. coli endotoxin in $0.1 \mathrm{ml}$ saline or $(O)$ or $0.1 \mathrm{ml}$ saline alone $(\square)$.

tion of $2.5 \times 10^{6 i}$ monocytes $/ \mathrm{ml}$. The cells were then incubated at $37^{\circ} \mathrm{C}$ in $5 \% \mathrm{CO}$, for 18 hours. After incubation, the cell suspensions were centrifuged for 15 minutes at $1000 \mathrm{~g}$ and the supernatant drawn off. A portion of the supernatant was heated to $70^{\circ} \mathrm{C}$ for 90 minutes to denature the IL-1. The IL-1 and heat-treated IL-1 were than stored at $-20^{\circ} \mathrm{C}$.

\section{Experiment I: Effect of ICV IL-I on Food Intake}

To determine if IL-1 can exert an anorexigenic effect when administered centrally, 6 rats were injected ICV with $20 \mu \mathrm{l}$ of human IL-1 and 6 with $20 \mu \mathrm{l}$ or heat-treated IL-1. Each injection was followed by $20 \mu$ lof artificial cerebrospinal fluid to clear the cannula of injectate. The rats were allowed a 24 hour period of recovery, and the experiment was repeated, switching the control and experimental injection groups in a crossover design for a total of 12 animals per group.

To determine whether placement of the cannula in the brain might prevent the anorexigenic effect of intraperitoneally administered IL-1, 6 cannula-bearing rats were injected IP with $1.5 \mathrm{ml}$ of IL- 1 and 6 were injected IP with heattreated IL- 1 .

\section{Experiment 2: Effect of ICV Endotoxin on Food Intake}

To determine if endotoxin itself might exert an anorexigenic effect when injected centrally, 12 rats were injected ICV with $10 \mathrm{ng} E$. coli endotoxin (0111:B4, Difco, Detroit) in $10 \mu \mathrm{l}$ of saline or $10 \mu \mathrm{l}$ of saline alone in a crossover design as described for Experiment 1. The rats were
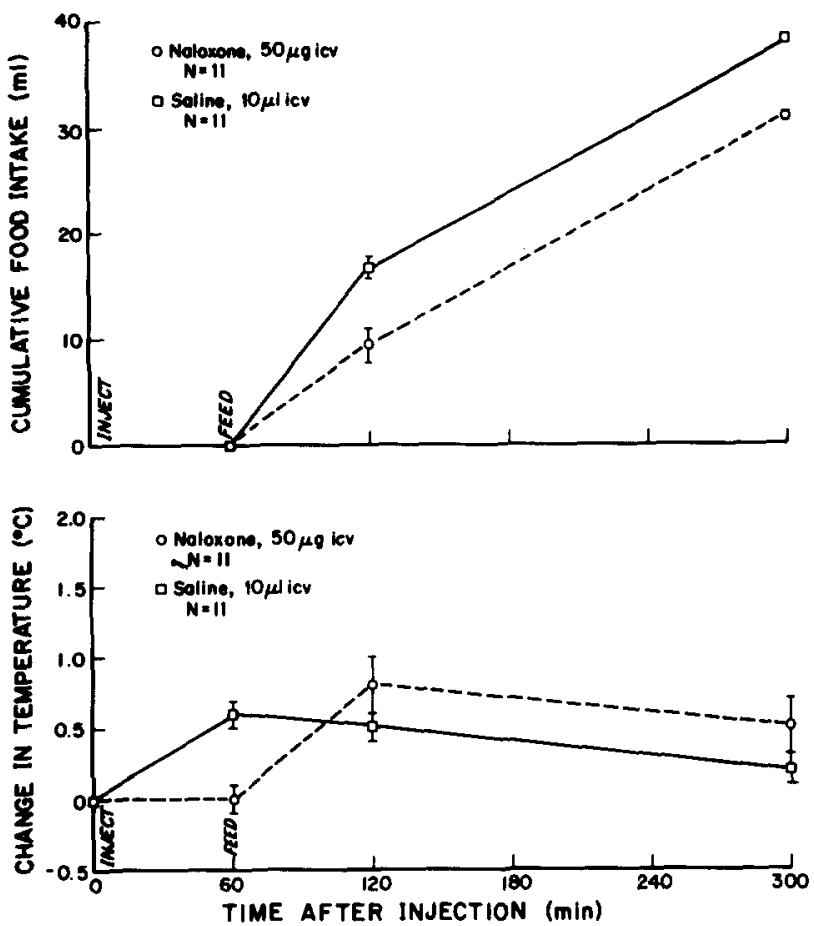

FIG. 4. Cumulative milliliters of food intake ( \pm S.E.M.) and change in body temperature $( \pm$ S.E.M. $)$ in fasted rats following intracerebroventricular injection of $50 \mu \mathrm{g}$ naloxone $\mathrm{HCl}$ in $10 \mu$ l saline (O) or $10 \mu \mathrm{l}$ of saline alone $(\square)$.

allowed a 48 hour period of recovery and were subsequently injected with $100 \mathrm{ng}$ of endotoxin in $10 \mu \mathrm{l}$ of saline or $10 \mu \mathrm{l}$ of saline alone. To ensure that the response to the endotoxin was not blunted by endotoxin tolerance, 6 randomly selected rats were injected intravenously (IV) with $100 \mathrm{ng}$ of endotoxin in $0.1 \mathrm{ml}$ of saline and 6 with $0.1 \mathrm{ml}$ saline alone.

\section{Experiment 3: Effect of ICV Naloxone $\mathrm{HCl}$ on Food Intake}

To validate that a centrally mediated suppression in food intake could be induced by a known anorexigenic agent using this animal model, 12 rats were injected ICV with $50 \mu \mathrm{g}$ of naloxone $\mathrm{HCl}$ (kindly provided by Endo Pharmaceuticals, Wilmington, DE) in $10 \mu \mathrm{l}$ of pyrogen-free saline (saline) and 12 were injected with $10 \mu \mathrm{l}$ of saline alone, as described by Thornhill et al. [18].

\section{RESULTS}

\section{Experiment 1: Effect of ICV IL-I on Food Intake}

As shown in Fig. 1 (bottom panel), injection of IL-1 into the lateral cerebroventricle of the rats caused a significant rise in body temperature by 60 minutes after injection compared with control animals injected with heat-treated IL-I (1.6 vs. $0.5^{\circ} \mathrm{C}, p=0.001$ ), validating activity of IL- 1 at the level of the hypothalamus. The mean change in body temperature over the five-hour experimental period was $1.7 \mathrm{vs}$. $0.6^{\circ} \mathrm{C}(p=0.001)$. In contrast, food intake of the rats injected ICV with IL-1 was not significantly different from control animals at any time during the four-hour feeding period (Fig. 
I, top panel). Repeated measures analysis of variance revealed no effect of injections on food intake $(p=0.6)$.

Intraperitoneal injection of IL-1 into rats implanted with ICV cannula significantly elevated body temperature by 120 minutes after injection compared to control animals injected with heat-treated $\mathrm{IL}-1\left(1.4 \mathrm{vs} .0 .5^{\circ} \mathrm{C}, p=0.006\right)$. The average change in body temperature over the 5 hour post-injection period was 1.0 vs. $0.5^{\circ} \mathrm{C}(p=0.02)$. Food intake of the cannula-bearing rats injected IP with IL-1 was reduced to $60 \%$ of controls (8.6 vs. $14.5 \mathrm{ml}, p=0.05)$ at 120 minutes after injections, similar to that found previously. These data indicate that the presence of the cannula did not interfere with the anorexigenic effect of peripherally administered $[L,-1$.

\section{Experiment 2: Effect of $1 \mathrm{CV}$ Endotoxin on Food Inake}

As shown in Fig. 2 (bottom panel), ICV injection of $10 \mathrm{ng}$ of endotoxin significantly elevated body temperature of the rats by 120 minutes after injection compared to salineinjected controls. The mean change in body temperature over the five-hour experimental period was 1.2 vs. $0.5^{\circ} \mathrm{C}$ $(p=0.001)$. Food intake of rats injected ICV with endotoxin (top panel) was not significantly different from controls at any time, with no treatment effect evident using repeated measures analysis of variance $(p=0.2)$. Similar data were obtained following ICV injection of $100 \mathrm{ng}$ of endotoxin (data not shown). When these same rats were injected IV with 100 ng of endotoxin (Fig. 3), body temperature was significantly elevated over the five-hour experimental period $(p=0.01)$. Repeated measures analysis of variance revealed a significant treatment effect on food intake of the endotoxin-injected rats compared to saline-injected controls $(p=0.05)$.

\section{Experimemt 3: Effeet of IC $V$ Naloxone HCl on Fond Intake}

As shown in Fig. 4, injection of $50 \mu \mathrm{g}$ naloxone $\mathrm{HCl}$ into the lateral cerebral ventricle of the fasted rats did not significantly alter body temperature over the five-hour experimental period compared to saline-injected control animals $\left(0.5\right.$ vs $\left..0 .2^{\circ} \mathrm{C}, p=0.3\right)$. Repeated measures analysis of variance revealed that $\mathrm{ICV}$ injection of naloxone $\mathrm{HCl}$ did reduce mean hourly food intake of the fasted rats compared with saline-injected controls $(p=0.001)$ indicating that anorexia could be centrally induced using this model.

\section{DISCUSSION}

In the present study, ICV injection of IL-1 did not affect food intake of the fasted rats at any time during the four-hour feeding period. One possible explanation for this negative finding might be that the presence of the ICV cannula somehow influenced brain function so as to prevent a response to anorexigenic stimuli in general. This possibility was ruled out by the demonstration that ICV-administered naloxone did reduce food intake. Accordingly, we conclude that IL-1 does not affect food intake at a central nervous site bathed by the cerebrospinal fluid.

We have previously shown that an intravenous injection of $l:$ roli endotoxin significantly reduces food intake of fasted rats $[13,14]$. While endotoxin is a potent stimulus for IL-1 production by peripheral monocytes [1]] and by brain tissue [8], it is possible that endotoxin-induced anorexia is not mediated centrally by $1 \mathrm{~L}-1$, but by endotoxin irself $[3,16]$. In the present study. ICV injection of both 10 and 100 ng endotoxin resulted in a significant elevation in body temperature, but neither dose of endotoxin affected food intake of the fasted rats compared to saline-injected controls. However, IV injection of $100 \mathrm{ng}$ of endotoxin significantly suppressed food intake compared with saline-injected controls. We conclude that anorexigenic effects of endotoxin are not mediated at a central nervous site bathed by the cerebrospinal fluid.

Based on Brobeck's theory of the thermostatic regulation of food intake [5], it had been postulated that fever may be a factor in the suppression of food intake during infection. Baile $' t a l$. [1] found that when the antipyretic drug dipyrone was administered to sheep simultaneously with endotoxin, fever was blocked and food intake was improved for a short while compared to endotoxin-injected animals that did not receive the antipyretic. McCarthy it al. [13] found that injection of sodium salicylate to lower endotoxin-induced fever in rats did not eliminate the endotoxin-induced suppression of food intake; however, the sodium salicylate was not administered until after the onset of the fever, making it difficult to rule out a possible initial effect of fever on food appetite. In the present study, ICV injection of IL-I caused a large rise in body temperature before the rats were fed, but did not affect food intake of the fasted rats at any time during the four-hour feeding period compared to the control animals. Accordingly, we conclude that the onset of fever does not contribute to the suppression of food intake following peripheral injection of IL- 1 or endotoxin.

In summary, the findings of the present study indicate that neither endotoxin or IL-1 acts centrally to reduce food appetite nor do they affect food intake indirectly by inducing fever. What might be the peripheral mechanism of action? Turner and Berry [19] reported that IP injection of endotoxin inhibited gastric emptying in mice within 5 minutes after injection. Leek and van Miert [12] reported that IL- 1 is detectable in the plasma of sheep during endotoxin-induced gastric stasis. Van Miert and van Duin [20] found that injection of leukocyte supernatant containing IL-1 reduced gastric motility of goats to about $60 \%$ of controls by 60 minutes after injection. This effect did not occur if the supernatant was heated to $75^{\circ} \mathrm{C}$ for 30 minutes prior to injection. These findings suggest that IL-I might indirectly affect food intake via effects on the rate of gastric emptying and the development of gastric distention [21].

\section{REFERENCES}

1. Baile, C. A., J. Naylor, C. L. McLaughlin and C. A. Catanzano Endotoxin elicited fever and anorexia and elfazepam-stimulated feeding in sheep. Physiol Behav 27: 271-277, 1981.

2. Bailey, P. T., F. B. Abeles, E. C. Hauer and C. A. Mapes. Intracerebroventricular administration of leukocytic endogenous mediators in the rat. Proc Soc Exp Biol Med 153: 419-423, 1976.

3. Bennett, I. L., R. G. Petersdorf and W. R. Keene. Pathogenesis of fever: evidence for direct cerebral action of bacterial endotoxins. Trans Assoc Am Physicians 70: 64-73, 1957.
4. Blatteis, C. M. Newer putative central neurotransmitters: Role in thermoregulation. Fed Proc 40: 2735-2740, 1981.

5. Brobeck, J. R. Models for analysing energy balance. In: Body Weight Regulatory System: Normal and Disturbed Mechanisms, edited by L. A. Cioffi, W. P. T. James and T. B. Van Ittalie. New York: Raven Press, 1981, pp. 1-9.

6. Clark, W. G. and J. M. Lipton. Brain and pituitary peptides in thermoregulation. Pharmacol Ther 22: 249-297, 1983.

7. Dinarello, C. A. Interleukin-1 and the pathogenesis of the acute phase response. $N$ Engl J Med 311: 1413-1418, 1984. 
8. Fontana, A., E. Weber and J. M. Dayer. Synthesis of interleukin-1/endogenous pyrogen in the brain of endotoxin treated mice. J Immunol 133: 1696-1698, 1984.

9. Hamilton, C. L. Interactions of food intake and temperature regulation in the rat. J Comp Physiol Psychol 56: 476-488, 1963.

10. Jones, P. G., C. A. Kauffman, A. G. Bergman, C. M. Hayes, M. J. Kluger and J. G. Cannon. Fever in the elderly: production of leukocytic pyrogen by monocytes from elderly people. Gerontology 30: 182-187, 1984.

11. Lachman, L. B. Interleukin-1: release from LPS-stimulated mononuclear phagocytes. In: Beneficial Effects of Endotoxin, edited by A. Nowotny. New York: Plenum Press, 1983, pp. 283-305.

12. Leek, B. F. and A. S. J. P. A. M. van Miert. An analysis of the pyrogen induced inhibition of gastric motility in sheep.J Physiol (Lond) 215: 28P-29P, 1971.

13. McCarthy, D. O., M. J. Kluger and A. J. Vander. The role of fever in appetite supression after endotoxin administration. Am J Clin Nutr 40: 310-316, 1984.

14. McCarthy, D. O., M. J. Kluger and A. J. Vander. The role of interleukin-1 in the suppression of food intake during infection. Am J Clin Nutr 42: 1179-1182, 1985.

15. Morley, J. E., A. S. Levine, B. A. Gosnell and C. J. Billington. Neuropeptides and appetite: contribution of neuropharmacological modeling. Fed Proc 43: 2903-2907, 1984.
16. Ogawa, Y. and S. Kanoh. Involvement of central action of lipopolysaccharide in pyrogen fever. Jpn J Pharmacol 36: 389. $395,1984$.

17. Powanda, M. C. and W. R. Beisel. Hypothesis: leukocyte endogenous mediator/endogenous pyrogen/lymphocyte activating factor modulates the development of nonspecific and specific immunity and affects nutritional status. Am J Clin Nutr 35: $762-768,1982$.

18. Thornhill, J. A., B. Taylor, W. Marshall and K. Parent. Central, as well as peripheral naloxone administration suppresses feeding in food-deprived Sprague-Dawley and genetically obese (Zucker) rats. Physiol Behav' 29: 841-846, 1982.

19. Turner, M. M. and L. J. Berry. Inhibition of gastric emptying in mice by bacterial endotoxin. Am J Physiol 205: 113-116, 1963.

20. van Miert, A. S. J. P. A. M. and C. T. M. van Duin. The effects of bacterial pyrogens and leucocytic pyrogen upon gastric motility and heart rate frequency in conscious goats. Zentralbl Veterinarmed [A] 21: 692-702, 1974.

21. Wirth, J. B. and P. R. McHugh. Gastric distension and short term satiety in the rhesus monkey. Am J Physiol 245: R174 R180, 1983. 